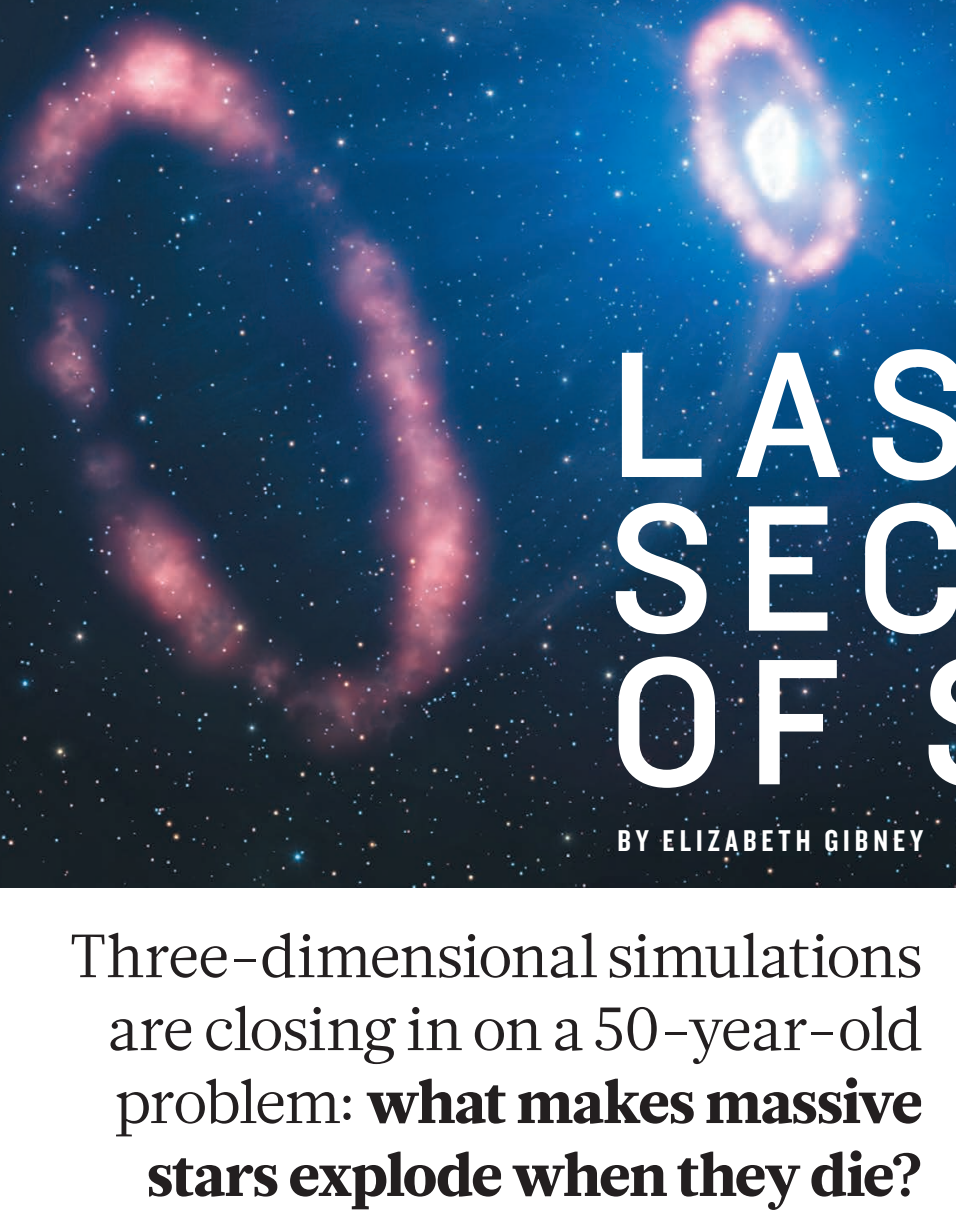

A

fter spending three months trying to blow up a star, Hans-Thomas Janka and his team finally saw what they had been waiting for. Like the world's most patient pyromaniacs, they watched their massive stellar simulation - rendered in painstaking detail — inch closer to detonation. Each day, their supercomputer ticked through just 5 milliseconds of the star's life.

But perseverance has its rewards. In the team's previous attempts to make a realistic simulation, the stellar fireworks always petered out. This time, in 2015, Janka watched as the shock wave needed to drive the explosion continued to grow; the mock star was going supernova ${ }^{1}$."That was the moment we recognized that, $\mathrm{OK}$, now we are at the point we longed to be at for two decades," says Janka, a theoretical astrophysicist at the Max Planck Institute for Astrophysics in Garching, Germany. "We were on the path to clarifying the explosion mechanism of these massive stars."

For more than half a century, physicists have suspected that the heat produced by elusive particles called neutrinos, created in the core of a star, could generate a blast that radiates more energy in a single second than the Sun will in its lifetime. But they have had trouble proving that hypothesis. The detonation process is so complex - incorporating general relativity, fluid dynamics, nuclear and other physics - that computers
An artist's impression of supernova 1987A, showing its asymmetric ejection of material. have struggled to mimic the mechanism in silico. And that poses a problem. "If you can't reproduce it," Janka says, "that means you don't understand it."

Now, improvements in raw computing power, along with efforts to capture the stellar physics in acute detail, have enabled substantial progress. Janka's simulation marked the first time that physicists had been able to get a realistic 3D model of the most common type of supernova to explode. Just months later, a competing group based at Oak Ridge National Laboratory in Tennessee repeated the feat with a heavier, more complex star ${ }^{2}$. The field is now buzzing, with more than half a dozen teams currently working on exploding stars in 3D. Many researchers are confident that they are closing in on identifying the ingredients that are crucial to generating such blasts.

The effort faces challenges. Three-dimensional models are still in their infancy and vary widely — and simulated stars sometimes still fail to blow. Time is also of the essence. Stellar explosions beyond the Milky Way are a common sight, but astronomers want to see one up close, in our own backyard. One or two are expected to happen every century, and the next one could occur at any time. When it does, astronomers will be equipped to see more than just the light emanating from the outer layers of the explosion. They will be able to use state-of-the-art detectors to pick up gravitational waves and neutrinos emanating from the centre of the blast. Not only can predictions from simulations help astronomers to tailor their instruments to best capture the explosion, but they will also be essential for making sense of the data. "My goal is to have the models sufficiently sophisticated so that when a Galactic supernova goes off, we're ready for it," says Anthony Mezzacappa, who leads the Oak Ridge team.

\section{BEHIND THE SHOCK}

When a star between around 8 and 40 times the mass of the Sun comes to the end of its life, it tends to go out with a bang, releasing more energy than one trillion trillion nuclear warheads. These "core collapse" explosions 


\section{EXPLODING A VIRTUAL STAR}

When a massive star dies, a neutron star can form at the centre of its iron core. Infalling iron hits this ultra-dense orb and rebounds, creating a shock wave. In simulations such as this one, of a 20 -solar-mass star, the crucial moments occur right after the bounce.
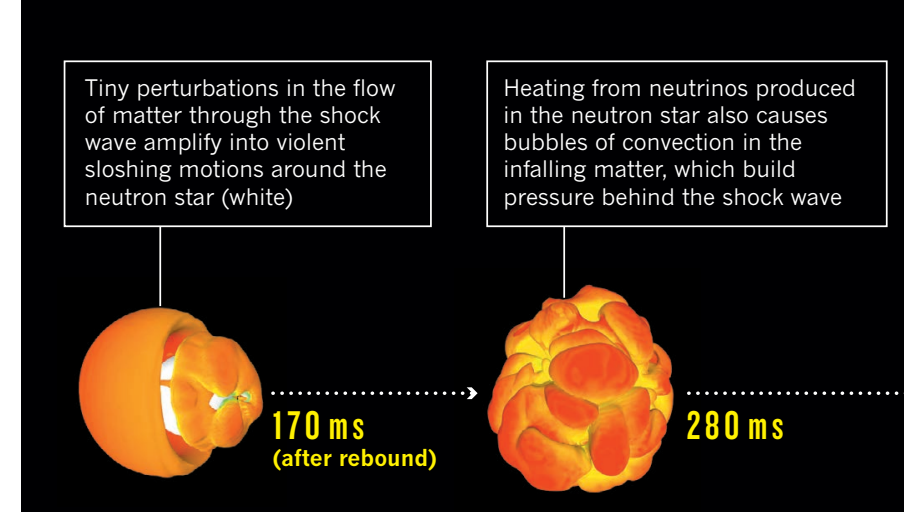

\section{(1)}
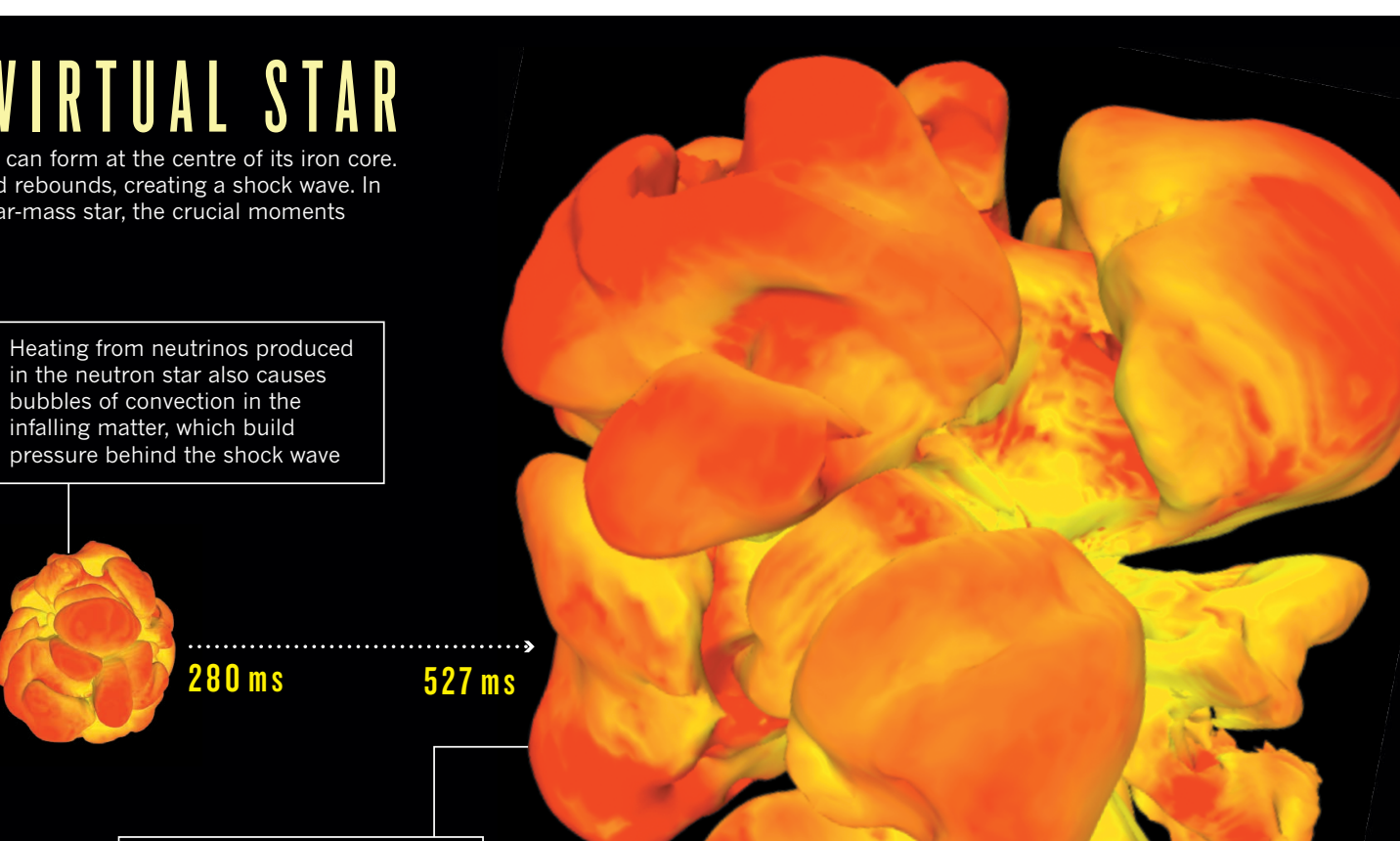

RELATIVE VELOCITY OF MATTER

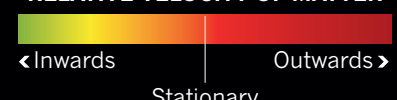

Heat from neutrinos and the pressure of these motions eventually drive the shock wave rapidly out into layers beyond the core - the star is exploding

pressure. Getting to the state of today's simulations, and the still-tentative explanations of how core-collapse explosions happen, is a decades-long story of increasing complexity that began with something that looked little like a star: simple 1D models.

Although fairly crude, those models revealed the first vital ingredient of a core-collapse supernova: the neutrinos produced through particle interactions in the newly formed neutron star. Neutrinos, which are nearly massless, barely engage with other particles. But in 1966, theorists calculated that if even a tiny fraction of their energy was absorbed by the dense matter around the core, the heat would be enough to rekindle the shock wave and drive it out ${ }^{4}$. Evidence in favour of the idea might have been bolstered by a lucky break. In 1982, computational physicist James Wilson, then at Lawrence Livermore National Laboratory in Livermore, California, left a simulation to run overnight — some say accidentally. He returned to find that, after a delay, enough neutrinos had diffused out of the neutron star to heat matter behind the shock wave and drive it out of the star. Until then, physicists had not realized that a stalled wave could be revived. "If models were not run to such late times, we would not have seen it," says Mezzacappa.

Neutrino heating became the field's main focus of research, but the more detailed the simulations and the larger the mass of their starting star, the less often modellers saw explosions. Although neutrinos pushed the stars close to the brink, it became clear that they needed a helping hand.

\section{FULL FIREWORKS}

The first clue as to what might provide the boost came in 1987, when astronomers observed a supernova in a nearby galaxy — the Large Magellanic Cloud. At the time, 1D models necessarily assumed stars were perfect spheres, made up of concentric layers of fusing elements and containing dynamics that could be captured with just one coordinate: distance from the centre. But the intermingled way that supernova 1987A spewed out elements suggested that layers must mix, a dynamic process that would be impossible to describe in one dimension.

With the advent of much more powerful computers in the 1990s, modellers were able to capture this motion by progressing from $1 \mathrm{D}$ to $2 \mathrm{D}$ simulations. In two dimensions, neutrino heating acted like a stove flame ticle physics of neutrinos and the behaviour of matter under extreme 
under a pan of water, creating convection and turbulence that churned up fresh matter for the particles to heat, boosting the pressure behind the shock wave. And in 2003, Mezzacappa's team found that perturbations in the shock wave can grow rapidly into large sloshing motions and violent rotations - known as standing accretion shock instability (SASI). These motions charge the shock wave and help the star to explode.

Still, physicists worried that the compromises they made in rendering stars in two dimensions might artificially boost the chance of explosion. Indeed, when computing power made crude 3D models feasible in the early 2010s, the models were once again "reluctant to explode", says Bernhard Müller, a computational astrophysicist at Monash University in Melbourne, Australia, who was part of Janka's team until 2014. It was not until the advent of faster supercomputers in 2012 that researchers began to be able to weave together general relativity and detailed nuclear and particle physics to get 3D stars to start to blow, in models that ran from scratch.

Reaching that milestone lends confidence to the assumption that neutrino heating, convection and SASI oscillations are behind the explosions, says Janka. Since 2015, teams around the world - including groups at California Institute of Technology (Caltech) in Pasadena, Princeton University in New Jersey, Michigan State University and Fukuoka University in Japan - have begun to work on 3D models. A substantial fraction of those simulations end in explosions (see 'Exploding a virtual star'). The trend will need to continue across a range of stars of different masses and initial structures to prove that physicists understand the mechanism, but Müller is optimistic. "We seem to be converging towards a solution for this problem of shock revival," he says.

Others are more sceptical. Shock waves emerge more easily in relatively small stars. When Janka's team attempted to explode a larger 3D star in 2015 - one that was 20 rather than 10 times the mass of the sun - they succeeded only because they pushed one interaction rate for neutrinos to the lowest level that the error bars from particle physics would allow. Today's simulations, which use more realistic initial conditions, still sit uncomfortably close to the tipping point between exploding and sputtering out, and no one is quite sure why. "In nature, these things explode robustly all the time," says Couch. The models' reluctance to do so is "probably telling us either we're not doing it accurately enough with the physics we are including, or we're missing physics".

A solution is to keep building richer models. But on today's supercomputers - which perform the equivalent of tens of thousands of stateof-the-art home computers running at once - this process still takes months, and modellers must necessarily make approximations and simplifications. Upgrades due in the next few years to supercomputers in the United States, Europe and Japan would cut the run time for a 3D explosion down to weeks. But even after that, computers would need to be made 100 times more powerful to churn through a 3D simulation that takes into account the full complement of physics, says Mezzacappa. Such computers could be another decade away, he says.

In the meantime, physicists are focusing on adjusting their models to see whether they can work out how the three main ingredients - neutrino heating, convection and SASI oscillations - interact, and whether any others might be missing. Some are exploring whether rotation and magnetic fields might help fuel the explosion. Others are basing models on more-realistic stars, with perturbations built in from the start. But comparing across simulations is difficult. Each group's models include not just different physics, but different shortcuts, resolution and pixel geometry - all of which can affect the result. And teams defend their choices fiercely. "I would go to conferences and people from different groups were almost fighting with each other, each saying 'my code is better," says Modjaz. "There was no way to tell, because they wouldn't publish their codes or compare them in a regular fashion."

Now groups are realizing that to make progress, they might need to find ways to make those comparisons, says Modjaz. A new generation of modellers, including Couch and Evan O'Connor at Stockholm University, have pioneered the publication of codes and encouraged others to do the same. Janka advocates creating a set of standardized test problems, with the same well-defined ingredients and initial conditions, to be used by the whole

field. "I think it will be a next very important step for the community, to enhance its credibility and the reliability of the results put out," he says.

\section{CORE QUESTIONS}

The true test will be whether these explosions actually resemble the ones in nature. Models are now sophisticated enough — and computing capacity is great enough - to run simulations beyond the first fraction of a second after the shock wave forms to when the blast wave ultimately breaks through the surface of the star many hours later. The predictions of supernovae shape, energy and chemistry generated by such models can then be compared with a real star's exploding outer layers, as well as with the motion of the leftover core.

But studying light from the star's surface - as well as ghostly remains that linger for centuries - can give only limited information about the explosion. "It's like going to a dermatologist to ask about your heart," says Couch. Neutrinos and gravitational waves, which pass through matter relatively unimpeded, could allow astronomers to see deep inside the star. In 1987, three neutrino detectors picked up 25 neutrinos emitted from supernova 1987A. In the decades since, subsequent detectors - such as IceCube at the South Pole and Super-Kamiokande in Japan - have been built that could be sensitive to tens of thousands of neutrinos emitted by a nearby supernova. When the neutrinos of such an explosion reach Earth, their energy, abundance and emission rate could reveal, for example, roughly how massive and how compact the neutron star is, as well as how much mass it continued to accrete after collapse. Any SASI wobble would cause neutrino emissions to rise and fall, and be visible as oscillations in the signal. "You could have a direct smoking gun for what's going on inside the supernova," says Müller.

The value of detecting a supernova through its neutrinos is so great that upgrades to IceCube are

"I think if you pushed lots to tell the truth, we just really like blowing things up." usually done on only part of the detector at a time, so that it won't miss a once-in-a-lifetime event. The youngest supernova remnant found so far in our Galaxy is about 150 years old, but researchers say that it would be a statistical fallacy to think the next explosion is 'overdue. "No one can tell you when it will take place, so you have to be alert all the time," says Janka.

If astronomers get lucky, the Laser Interferometer Gravitational-Wave Observatory (LIGO) in the United States and its sister observatory Virgo near Pisa, Italy, should also be able to observe the blast, although the signal is not expected to be as clear as those of the black-hole and neutron-star mergers found so far. Sarah Gossan, a physicist at Caltech and a member of the LIGO team, says that simulations will be needed to help find a faint signal among the noise and to decipher the information it contains. "We'll be able to inform our simulations from our observations, and vice versa," Gossan says.

To prepare for such events, modellers such as Janka will need to simulate dozens of different 3D stars. In October, his team lit the fuse on a particularly complex model - a 19-solar-mass star, whose final minutes they had also modelled so that they could begin the collapse under conditions as messy and realistic as possible. They won't find out until at least July whether it will blow or not. But "by now", he says, "we're pretty used to being patient".

\section{Elizabeth Gibney is a senior reporter for Nature based in London.}

1. Melson, T., Janka, H.-T. \& Marek, A. Astrophys. J. 801, L24 (2015).

2. Lentz, E. J. et al. Astrophys. J. 807, L31 (2015)

3. Burbidge, E. M., Burbidge, G. R., Fowler, W. A. \& Hoyle, F. Rev. Mod. Phys. 29 547-650 (1957).

4. Colgate, S. A. \& White, R. H. Astrophys. J. 143, 626-681 (1966) 